\title{
Successful Treatment of Osgood-Schlatter Disease with Autologous-Conditioned Plasma in Two Patients
}

\author{
Dirk-Jonas Danneberg ${ }^{1}$ \\ ${ }^{1}$ Department of Orthopaedics and Sports Medicine, \\ Darmstadt, Germany
}

Joints 2017;5:191-194.
Address for correspondence Dirk-Jonas Danneberg, MD, Department of Orthopaedics and Sports Medicine, Nieder Ramstädter Street 160, 64285 Darmstadt, Germany (e-mail: info@orthopaedy.com).

\author{
Abstract \\ Keywords \\ - Osgood-Schlatter \\ disease \\ - autologous- \\ conditioned plasma \\ - platelet concentrate \\ - patellar ligament \\ - apophysitis
}

Osgood-Schlatter Disease (OSD) is a painful, growth-related overuse condition of the tibial tuberosity, leading to inflammation of the patellar ligament at the tibial tuberosity. It primarily affects young adolescents, athletic population, and usually, resolves with age or skeletal maturity. Therapy is usually conservative, with surgery indicated in a minority of cases. For patients with treatment-resistant or refractory OSD, an alternative is the application of autologous platelet concentrate. Here, we describe two cases in which autologous-conditioned plasma therapy was used to treat OSD, and present the treatment protocol developed in our clinic.

\section{Introduction}

Osgood-Schlatter disease (OSD) is a painful, growth-related overuse condition of the tibial tuberosity in which inflammation of the patellar ligament at the tibial tuberosity occurs. ${ }^{1,2}$ OSD, a traction apophysitis of the anterior aspect of the tibial tuberosity (ATT), is caused by quadriceps muscle contractions at the proximal tibial apophysis insertion. This leads to small avulsion fractures, ${ }^{1,2}$ including partial traumatic avulsion of the tibial tuberosity at the patellar tendon insertion. Trauma to the ATT can also lead to acute OSD. ${ }^{3}$ Treatment with analgesics, physiotherapy, and reduction of physical activity is recommended. $^{3}$ However, the duration of symptoms can be lengthy. ${ }^{4,5}$ Injections of autologous-conditioned plasma (ACP) are increasingly being used for a variety of musculoskeletal conditions. ${ }^{6-10}$ The growth factors contained in ACP are thought to influence mechanisms essential for tissue repair (e.g., modulation of inflammatory processes, chemotaxis, cell proliferation and migration, and matrix synthesis and differentiation). ${ }^{11-14}$ Therapeutic application of these treatments is safe and minimally invasive, ${ }^{15}$ and the preparation of conditioned plasma from autologous blood is simple. Here, we describe a case of OSD presenting in a young adult male after resolved childhood OSD and a case of treatment-resistant OSD in an adolescent male.

\section{Methods}

\section{Case 1}

A 23-year-old male tennis player with a childhood history of OSD resolving with age presented with right knee pain after a direct frontal impact onto the head of the tibia. On examination, he had tenderness at the tibial tuberosity and a small effusion, but no overlying erythema or limited range of motion. Knee X-rays demonstrated patellar tendon edema, and a sliver-like osseous density anterior to the apophysis of the tibial tuberosity, confirming the diagnosis of OSD. Sonographic imaging revealed a fluid layer or inflammatory fluid collection around the completely healed apophysis. Gait analysis revealed internal rotation of the lower leg.

\section{Case 2}

A 14-year-old male patient presented with reoccurring bilateral knee pain from persisting treatment-resistant OSD. He first presented with knee pain at the age of 12 , and played tennis 5 to 6 times/week. Previous repeated therapeutic interventions included extended resting phases, orthopedic insoles, physiotherapy, ultrasound therapy, and pulsed magnetic field therapy. Due to the existing diagnosis of treatmentresistant OSD, only confirmatory sonography and magnetic resonance imaging (MRI) scans were performed (- Fig. 1). published online August 24, 2017
DOI https://doi.org/ 10.1055/s-0037-1605384. ISSN 2282-4324.
Copyright @ 2017 Georg Thieme Verlag KG Stuttgart · New York

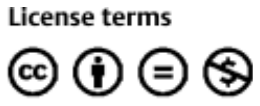




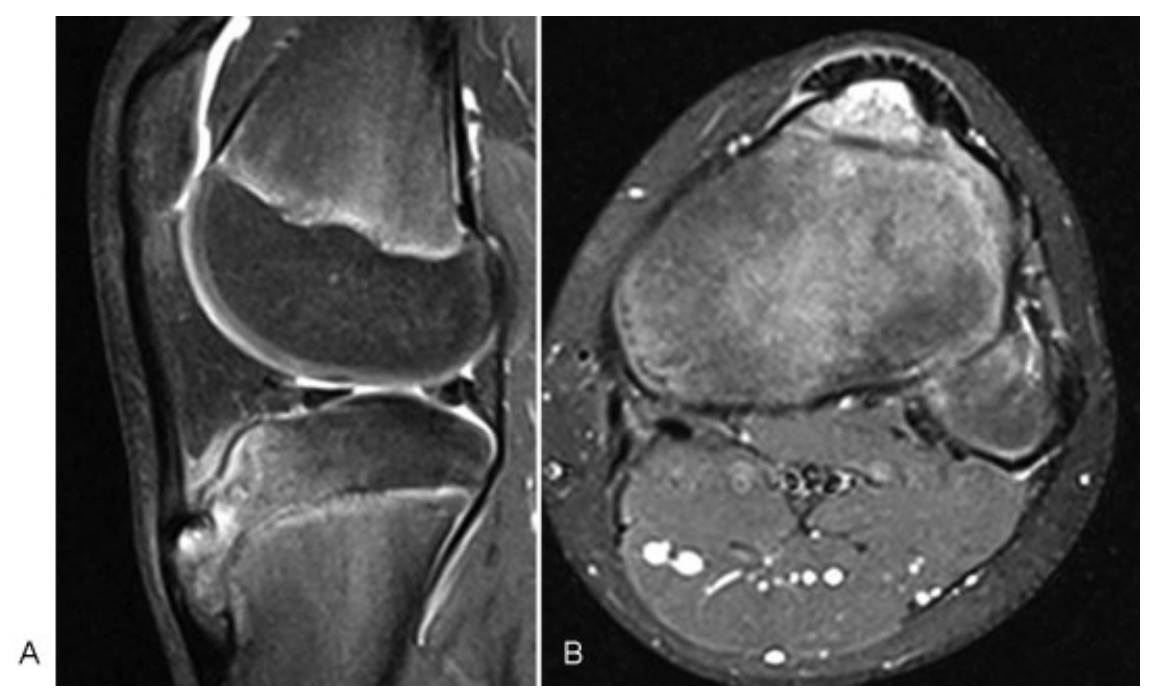

Fig. 1 Magnetic resonance image of the 14-year-old patient (case 2): (A) Sagittal scan; (B) axial scan.

\section{Treatment}

The standard ACP treatment protocol used at our clinic was applied. Patients received once-weekly subcutaneous injections of $1 \mathrm{~mL} \mathrm{ACP}$ on either side of the palpable OsgoodSchlatter lesion/swelling, for a total of three to five applications. ${ }^{16}$ The first patient, was treated in the right knee for 3 weeks, and the second patient in both knees for 4 weeks. The Arthrex ACP Double Syringe (Arthrex GmbH, Munich, Germany) was used, which uses a sterile double syringe system to prepare a leukocyte-poor but platelet-rich ACP. Around $15 \mathrm{~mL}$ of blood drawn into the double syringe produced about $5 \mathrm{~mL}$ of ACP. Centrifugation at 1,500 rpm for 5 minutes separated erythrocytes from the plasma components. The top portion of the plasma was drawn up into the inner syringe, without disrupting the erythrocyte layer, and used for injection. No activating agent was added. The knee was held in extension during injection to relax the tendon fibers, facilitating ACP diffusion into the injured area. Corticosteroids and local anesthesia were not administered. ${ }^{17}$ Cryotherapy was used for pain relief, with an elastic zincpaste cooling bandage application (Nika Medical Produkte $\mathrm{GmbH}$, Krefeld, Germany) for 24 hours after every injection, and self-administered treatment with an ice popsicle, two to three times daily for 15 minutes. The knee was bandaged twice daily using TRAUMA RÖD 301 Physiko Balsam K (Pharma-Biologica GmbH, Worms, Germany). During the day, patients wore a PUSH Patella Brace (OFA, Bamberg, Germany) to reduce the traction force on the lower leg until 6 months after resolution of pain. At night, kinesiology tape was used to bandage across the tibial head. Ibuprofen, $200 \mathrm{mg}$ daily for 10 days, and Wobenzym Plus (Mucos Pharma GmbH \& Co. KG, Berlin, Germany) orally (3-0.3) for 4 weeks were prescribed. The patients underwent physiotherapy and gait optimization to correct foot malposition and received dynamic shoe insoles. Targeted training during the injection therapy was prohibited. The sonographic evaluation was performed before, during, and after the conclusion of the ACP injection therapy.

\section{Results}

There were no postinjection complications. The first patient experienced a subjective pain reduction of approximately $50 \%$ after one injection. After 3 weeks, the patient was painfree and able to return to sport. The second patient was painfree after 6 weeks and able to return to sports; he has not experienced a relapse in OSD since treatment.

\section{Discussion}

OSD usually resolves with age or conservative therapy (limited physical activity and mild analgesia), though it can be treatment-resistant or reoccurring. Both patients described here were active and intensive tennis players, fitting the typical profile for OSD. For both patients, the novel ACP treatment came after the failure of conventional therapies. Shortly after treatment initiation, both patients had symptom resolution and could return to sport. In addition to the two described patient cases, four more patients were successfully treated with ACP in our clinic at the same time. However, the data collection was incomplete. None of the patients have reported a relapse in OSD, or any adverse events as a result of the ACP injections, and all have returned to sports. We recommend that patients undergo X-ray imaging, MRI, and sonography to confirm the diagnosis of OSD and to exclude additional underlying pathology. All six patients treated with ACP for OSD exhibited internal rotation upon gait analysis, and a tendency to skew or splayfoot. Thus, a digital, dynamic gait analysis should be performed on patients with OSD, and orthopedic insoles fitted when necessary. We have not identified other reported cases of ACP treatment for OSD; however, the Arthrex ACP Double Syringe system has been successfully used to prepare ACP for use in treatment-resistant patellar tendinopathy. ${ }^{6}$ In contrast to this study, we did not administer injections under ultrasound guidance. Our patients undertook a comprehensive home-care plan with cryotherapy, patellar braces, bandaging, kinesiology taping, and were allowed to use 
analgesics, possibly influencing the positive outcome. The two cases presented herein describe the successful, novel use of ACP therapy for OSD, providing the first insights into an alternative therapy for patients with standard-treatment failure or recurrent OSD. Further studies of ACP therapy for OSD in a larger patient group are indicated to optimize the treatment protocol further.

Note

Patient consent has been obtained.

\section{Acknowledgments}

The authors would like to thank Lyndsey Kostadinov, Dr. Michael Furrer, and Dr. Juan M. Escobar-Restrepo from Medicalwriters.com (Zurich, Switzerland) for medical writing support (funded by Arthrex).

\section{References}

1 Caine D, DiFiori J, Maffulli N. Physeal injuries in children's and youth sports: reasons for concern? Br J Sports Med 2006;40(09):749-760

2 de Lucena GL, dos Santos Gomes C, Guerra RO. Prevalence and associated factors of Osgood-Schlatter syndrome in a populationbased sample of Brazilian adolescents. Am J Sports Med 2011;39 (02):415-420

3 Gholve PA, Scher DM, Khakharia S, Widmann RF, Green DW. Osgood Schlatter syndrome. Curr Opin Pediatr 2007;19(01):44-50

4 Gerulis V, Kalesinskas R, Pranckevicius S, Birgeris P. Importance of conservative treatment and physical load restriction to the course of Osgood-Schlatter's disease [in Lithuanian]. Medicina (Kaunas) 2004;40(04):363-369

5 Mital MA, Matza RA, Cohen J. The so-called unresolved OsgoodSchlatter lesion: a concept based on fifteen surgically treated lesions. J Bone Joint Surg Am 1980;62(05):732-739

6 Charousset C, Zaoui A, Bellaiche L, Bouyer B. Are multiple plateletrich plasma injections useful for treatment of chronic patellar tendinopathy in athletes? a prospective study. Am J Sports Med 2014;42(04):906-911

7 Kon E, Filardo G, Delcogliano M, et al. Platelet-rich plasma: new clinical application: a pilot study for treatment of jumper's knee. Injury 2009;40(06):598-603

8 Zayni R, Thaunat M, Fayard JM, et al. Platelet-rich plasma as a treatment for chronic patellar tendinopathy: comparison of a single versus two consecutive injections. Muscles Ligaments Tendons J 2015;5(02):92-98

9 Foster TE, Puskas BL, Mandelbaum BR, Gerhardt MB, Rodeo SA. Platelet-rich plasma: from basic science to clinical applications. Am J Sports Med 2009;37(11):2259-2272

10 Lebiedziński R, Synder M, Buchcic P, Polguj M, Grzegorzewski A, Sibiński M. A randomized study of autologous conditioned plasma and steroid injections in the treatment of lateral epicondylitis. Int Orthop 2015;39(11):2199-2203

11 Laudy AB, Bakker EW, Rekers M, Moen MH. Efficacy of plateletrich plasma injections in osteoarthritis of the knee: a systematic review and meta-analysis. Br J Sports Med 2015;49(10):657-672

12 Eppley BL, Woodell JE, Higgins J. Platelet quantification and growth factor analysis from platelet-rich plasma: implications for wound healing. Plast Reconstr Surg 2004;114(06):1502-1508

13 Andia I, Sánchez M, Maffulli Basic Science N. Molecular and biological aspects of platelet-rich plasma therapies. Oper Tech Orthop 2012;22:3-9

14 Kon E, Filardo G, Di Martino A, Marcacci M. Platelet-rich plasma (PRP) to treat sports injuries: evidence to support its use. Knee Surg Sports Traumatol Arthrosc 2011;19(04):516-527

15 Boswell SG, Cole BJ, Sundman EA, Karas V, Fortier LA. Platelet-rich plasma: a milieu of bioactive factors. Arthroscopy 2012;28(03): 429-439

16 Cerza F, Carnì S, Carcangiu A, et al. Comparison between hyaluronic acid and platelet-rich plasma, intra-articular infiltration in the treatment of gonarthrosis. Am J Sports Med 2012;40(12): 2822-2827

17 Carofino B, Chowaniec DM, McCarthy MB, et al. Corticosteroids and local anesthetics decrease positive effects of platelet-rich plasma: an in vitro study on human tendon cells. Arthroscopy 2012;28(05):711-719 